\title{
Das margens para o centro: Mudanças na pesquisa em fundamentos da mecânica quântica, 1950-1990 ${ }^{+*}$
}

Olival Freire Junior ${ }^{1}$

Instituto de Física - Universidade Federal da Bahia

Salvador - BA

\section{Resumo}

A pesquisa em fundamentos da teoria quântica experimentou mudanças dramáticas na segunda metade do século XX, tendo evoluído de uma posição marginal na agenda de pesquisa da física para uma posição muito bem valorizada. Nós examinamos o conjunto de fatores, internos e externos, que condicionaram esta mudança. Especial atenção foi prestada à atividade dos físicos que foram os protagonistas destas mudanças, os quais nós denominamos de dissidentes quânticos.

Palavras-chave: História da física quântica; Teorema de Bell; Dissidentes quânticos.

\begin{abstract}
Researches on the foundations of quantum mechanics have undergone dramatic changes after 1950. It evolved from a marginal stand in the Physics research agenda to the mainstream of the subject. We analyzed external and internal factors which shaped this change and paid special attention to the practice of the physicists who were their actors. We called theses physicists the quantum dissidents.
\end{abstract}

Keywords: History of quantum Physics; Bell's theorem; Quantum dissidents.

\footnotetext{
+ Este artigo é uma tradução de "From the margins to the mainstream: Foundations of quantum mechanics, 19501990," publicado em Annalen der Physik, 527(5-6), A47-A51, 2015. A seção 5 foi ampliada no artigo ora publicado. A tradução foi preparada com o auxílio de Giselle Ferraz.

* Recebido: maio de 2015.

Aceito: maio de4 2015.

1 E-mail: freirejr@ufba.br
} 
Artigo dedicado ao Prof. José Maria Filardo Bassalo em homenagem aos seus 80 anos de idade.

\section{Introdução}

As pesquisas sobre fundamentos da mecânica quântica finalizaram o século XX como um campo de pesquisa de intenso desenvolvimento, incluindo promessas de aplicações relacionadas com o processamento de informações. O prestigiado prêmio Wolf de Física, em 2010, foi conquistado por John Clauser, Alain Aspect e Anton Zeilinger, "por suas seminais contribuições conceituais e experimentais aos fundamentos da física quântica, especialmente por uma série de testes cada vez mais sofisticados das desigualdades de Bell ou ampliações destas, usando estados quânticos emaranhados" ${ }^{2}$. As desigualdades de Bell e seu efeito físico relacionado, ou seja, o emaranhamento quântico, sintetizam as mudanças históricas sofridas pelas pesquisas sobre a possibilidade de modificar a mecânica quântica através do recurso a variáveis adicionais (por isto ocultas), desenvolvidas na segunda metade do século XX.

No passado este assunto ocupava uma posição marginal na agenda da pesquisa em física, de fato considerado uma questão filosófica além dos limites da pesquisa científica. $\mathrm{Na}$ década de 1950, a reinterpretação da mecânica quântica proposta por David Bohm também foi considerada "análoga aos anjos que as pessoas introduziram na Idade Média para explicar as coisas," ou um problema pertencente "à filosofia da ciência, ao invés do domínio próprio da física". Mais adiante, no início da década de 1970, alguns físicos influentes ainda se perguntavam se essas pesquisas eram "física de verdade" ${ }^{4}$. Considerar questões de fundamentos da teoria quântica como dignas de investigação é, assim, um tipo de renascimento na história da física, uma vez que estas questões estiveram presentes quando da criação desta teoria física.

Essa mudança requer uma explicação histórica e pode trazer esclarecimentos sobre os modos de funcionamento da ciência recente. Eu apresentei, em livro publicado recentemente, uma análise dos diversos fatores que desempenharam um papel neste cenário, chamando seus principais atores de dissidentes quânticos ${ }^{5}$. Eles foram dissidentes no sentido de que desafiaram o senso comum entre os físicos em sua época, período esse onde as questões de fundamentos eram consideradas como já resolvidas, não havendo mais sentido prosseguir na sua investigação. Como afirmado por John Bell e por Michael Nauenberg, em 1966, “o físico típico acha que [questões de fundamentos] foram respondidas há muito tempo e que ele

\footnotetext{
${ }^{2}$ Wolf Fund Prize Announcement 2010, online: $<$ http://www.wolffund.org.il/index.php?dir=site\&page=winners\&cs=283\&language =eng $>$. 3

3 Isidor Rabi (apud David Bohm) e Albert Messiah, respectivamente, citados em O. Freire Junior, The Quantum Dissidents - Rebuilding the foundations of quantum mechanics 1950-1990, Springer, 2015, p. 40 e 67.

${ }^{4}$ Idem, p. 271.

5 Idem.
} 
as entenderá completamente bastando que possa dispensar 20 minutos para pensar sobre isso" ". Eu apresento neste breve artigo uma síntese dessa história.

\section{As questões conceituais}

Desde o princípio, a mecânica quântica trouxe questões conceituais prementes que requeriam investigações. Estas questões incluíam a possibilidade de completar a teoria quântica com variáveis adicionais, visando recuperar ou o realismo ou o determinismo. Essa possibilidade foi explorada por Einstein em 1935 através do experimento de pensamento conhecido como EPR e revisitada por Bohm no início de 1950. A interpretação causal de Bohm foi um sinal de que algo estava errado na prova matemática de von Neumann contra as variáveis adicionais, mas não foi amplamente compreendida desta forma no momento. Depois de 30 anos sem causar muito barulho, esta prova foi revisada por John Bell. Ele mostrou que havia um conflito factual entre as previsões da mecânica quântica e a premissa adotada por Einstein do realismo local. Desde o início da década de 1970, uma série de experiências, iniciadas por Clauser, posteriormente desenvolvida pelo Aspect, e atualmente explorada por Zeilinger, entre outros, levaram à confirmação das previsões da mecânica quântica para a correlação das polarizações de um par de fótons separados um do outro. Estes experimentos levaram ao reconhecimento do emaranhamento como uma propriedade física de sistemas quânticos espacialmente separados. No entanto, ainda hoje, os físicos se esforçam para entender o emaranhamento, bem como para controla-lo, em pesquisas sobre criptografia e informação quântica.

Outra difícil questão conceitual veio a ser conhecida como o problema da medição quântica. Até a segunda guerra mundial duas soluções estiveram disponíveis. Para Niels Bohr, dispositivos, para que possam ser usados como dispositivos de medição, devem ser descritos usando a linguagem natural enriquecida pelos conceitos da física clássica. John von Neumann, por outro lado, identificou duas evoluções diferentes dos estados quânticos, uma representada pela equação de Schrödinger e a outra representada pelo truque matemático de um operador, um operador de projeção, para descrever medições. A solução de Bohr era muito filosófica e a de von Neumann muito matemática. Assim, não é nenhuma surpresa que a partir da década de 50 os físicos tenham começado procurar uma solução física para o problema. Os estados relativos de Hugh Everett foram motivados por sua aversão a duas evoluções diferentes para estados quânticos. Eugene Wigner conjecturou sobre o papel da mente nos processos de medição e sugeriu a formulação de uma equação de Schrödinger não-linear. Na década de 60, mesmo Bohrianos comprometido, a exemplo de Léon Rosenfeld, procuraram por uma solução física para identificar irreversibilidade como a característica específica da medição

\footnotetext{
${ }^{6}$ Idem, p. 243.
} 
quântica e apoiaram o programa da "amplificação termodinâmica," uma abordagem formulada pelos físicos italianos Daneri, Loinger, e Prosperi .

Atualmente, o problema relacionado à medição quântica continua sem solução satisfatória do ponto de vista conceitual, mas aprendemos muita física ao longo do caminho. $\mathrm{O}$ problema estava relacionado com a questão da transição entre a descrição quântica e a descrição clássica, e ambas as questões estão no centro do que chamamos hoje de descoerência, processo físico através do qual estados quânticos perdem sua coerência, ou seja, o processo pelo qual um estado puro torna-se uma mistura. A compreensão da descoerência surgiu ao longo de caminhos diferentes e independentes e envolveu o trabalho de Heinz Dieter Zeh e Erich Joos, Anthony Leggett e Amir Caldeira, e Wojciech H. Zurek, entre outros.

A conexão entre a física quântica e a gravitação, o quarto dos grandes problemas conceituais, está longe de ser resolvida até hoje. A busca por essa conexão tem, no entanto, estimulado a pesquisa nos fundamentos da mecânica quântica. A carreira de Bryce DeWitt foi dedicada a esta pesquisa e por isso ele se tornou um defensor vocal de uma interpretação alternativa da mecânica quântica, a dos estados relativos de Everett, denominada por DeWitt como a interpretação de muitos mundos.

Questões filosóficas também motivaram os físicos a reconsiderar os fundamentos da física quântica. As questões mais destacadas, neste terreno, têm sido a busca da recuperação do determinismo e as tentativas de acomodar o realismo ao formalismo matemático da teoria quântica.

Em retrospecto, a mera listagem dessas questões, das quais surgiram o emaranhamento e descoerência, seria suficiente para explicar a mudança no estatuto profissional da pesquisa sobre fundamentos, mas isso seria uma leitura anacrônica desses eventos, pois estas questões nem sempre foram consideradas significativas pelo conjunto da comunidade dos físicos.

\section{Os dissidentes quânticos}

Apesar da importância que estas questões têm agora, elas poderiam ter adormecido por um longo tempo, se não fosse pelo papel desempenhado pelos físicos que trabalharam com as mesmas. Exemplos de problemas inativos na história da física são a natureza da inércia e a relação entre as massas inercial e gravitacional na época de Newton. Séculos depois, isso foi revisitado por Ernst Mach e mais tarde por Einstein, e se compreendeu que elas escondiam bons problemas científicos. Assim, as pesquisas sobre fundamentos da teoria quântica só foram adiante por conta dos muitos físicos independentes que apostaram suas carreiras nestes temas. Eles sustentaram a ideia de que havia uma boa física a ser feita concernente aos fundamentos da mecânica quântica. Dentre estes, cabe destacar os nomes de Bohm, Everett,

\footnotetext{
${ }^{7}$ Idem, pp. 156-161 e 175-179. Daneri, A., Prosperi, G. M., Loinger, A.: Nuclear Physics, 33(2), 297-319 (1962).
} 
Bell, Wigner, Abner Shimony, Clauser, Bernard d'Espagnat, Aspect, Zeh, Franco Selleri, Leggett, dentre outros.

Estes físicos adotaram uma posição profissional e cultural contrária à sabedoria predominante daquela época, a qual dizia que as questões de fundamentos da quântica já tinham sido resolvidas pelos pais fundadores da disciplina. Eu os chamo de dissidentes quânticos, utilizando uma metáfora relacionada com dissidentes políticos do século 20. No médio prazo, tantos os dissidentes da ordem política quanto os dissidentes quânticos obtiveram reconhecimento em seus respectivos contextos, assim como suas causas se tornaram amplamente conhecidas e respeitadas. No caso dos dissentes quânticos, ganharam reconhecimento na medida em que a pesquisa em fundamentos se tornou um tópico de pesquisa altamente valorizado na agenda da física. Os dissidentes quânticos também enfrentaram preconceitos profissionais contra a sua pesquisa. Bell, após a publicação de seus trabalhos seminais, que exibiram as falhas na prova de von Neumann e, após apresentar o teorema, hoje chamado pelo seu nome, em 1966, recebeu uma carta de Rosenfeld afirmando: "não preciso dizer-lhe que eu considero sua caça aos parâmetros ocultos um desperdício do seu talento" ${ }^{8}$. Conhecedor deste preconceito, a primeira pergunta de Bell ao jovem Aspect, o qual procurava conselhos para os experimentos que planejava, foi: você tem um emprego estável? Aspect tinha, e isso fez toda diferença. Alguns dos preconceitos contra os dissidentes foram altamente efetivos. Em verdade, alguns deles pagaram um alto preço profissional por sua dissidência. Dois casos ilustram isso.

No início da década de 1970, Clauser era um jovem físico promissor. Ele fez seu doutorado na Universidade de Columbia sob a orientação de Patrick Thaddeus, trabalhando em medições de micro-ondas de radiação cósmica de fundo e, em seguida, fez experimentos com o teorema de Bell. Juntamente com Shimony, Horne, e Holt, Clauser traduziu o teorema para testes em laboratórios reais. Ele realizou um dos primeiros experimentos e obteve uma corroboração das previsões quânticas, que foi um marco na física, considerando as técnicas limitadas disponíveis no momento. Ele desenvolveu ainda mais o teorema de Bell, replicou experimentos sobre o teorema, e participou dos debates na criação da óptica quântica. Apesar dessas realizações, Clauser nunca obteve uma posição permanente nas universidades americanas. Uma das razões é que chefes de departamentos em uma posição para contratá-lo duvidavam que o teorema de Bell fosse física de verdade ${ }^{9}$.

Aproximadamente na mesma época, Zeh estava trabalhando em física nuclear quando escreveu um artigo que pode agora ser considerado um precursor da abordagem sobre a descoerência. $\mathrm{O}$ artigo sugere que o conjunto de um sistema mais um dispositivo de medição não pode ser considerado um sistema fechado, assim aproximações são necessários ao usar a equação de Schrödinger para descrevê-lo. O artigo não foi aceito pelo Il Nuovo Cimento e pelo Die Naturwissenschaften. Naquele momento, o pesquisador sênior em Heidelberg, onde

\footnotetext{
${ }^{8}$ The Quantum Dissidents, op. cit., p. 243. 
Zeh trabalhava, era o ganhador do Prêmio Nobel J. H. D. Jensen. Ele resolveu pedir o conselho de Rosenfeld sobre o artigo de Zeh. A opinião de Rosenfeld não poderia ter sido mais devastadora: "Eu tenho todas as razões do mundo para supor que tal concentrado de disparates mais selvagens não estão sendo distribuídos ao redor do mundo com a sua bênção. Eu penso ser útil chamar sua atenção para esse infortúnio" ". Zeh não leu a carta no momento - só recentemente ela veio a público - mas percebeu que o ambiente profissional em Heidelberg havia mudado com relação a ele. Jensen francamente o informou que quaisquer outras atividades sobre aquele assunto iria encerrar sua carreira acadêmica. $\mathrm{O}$ artigo foi resgatado, com o apoio de Wigner, e finalmente publicado. No entanto, Zeh considerou que sua carreira tinha sido destruída e, portanto, ele poderia se dedicar ao assunto proibido: os fundamentos da física quântica.

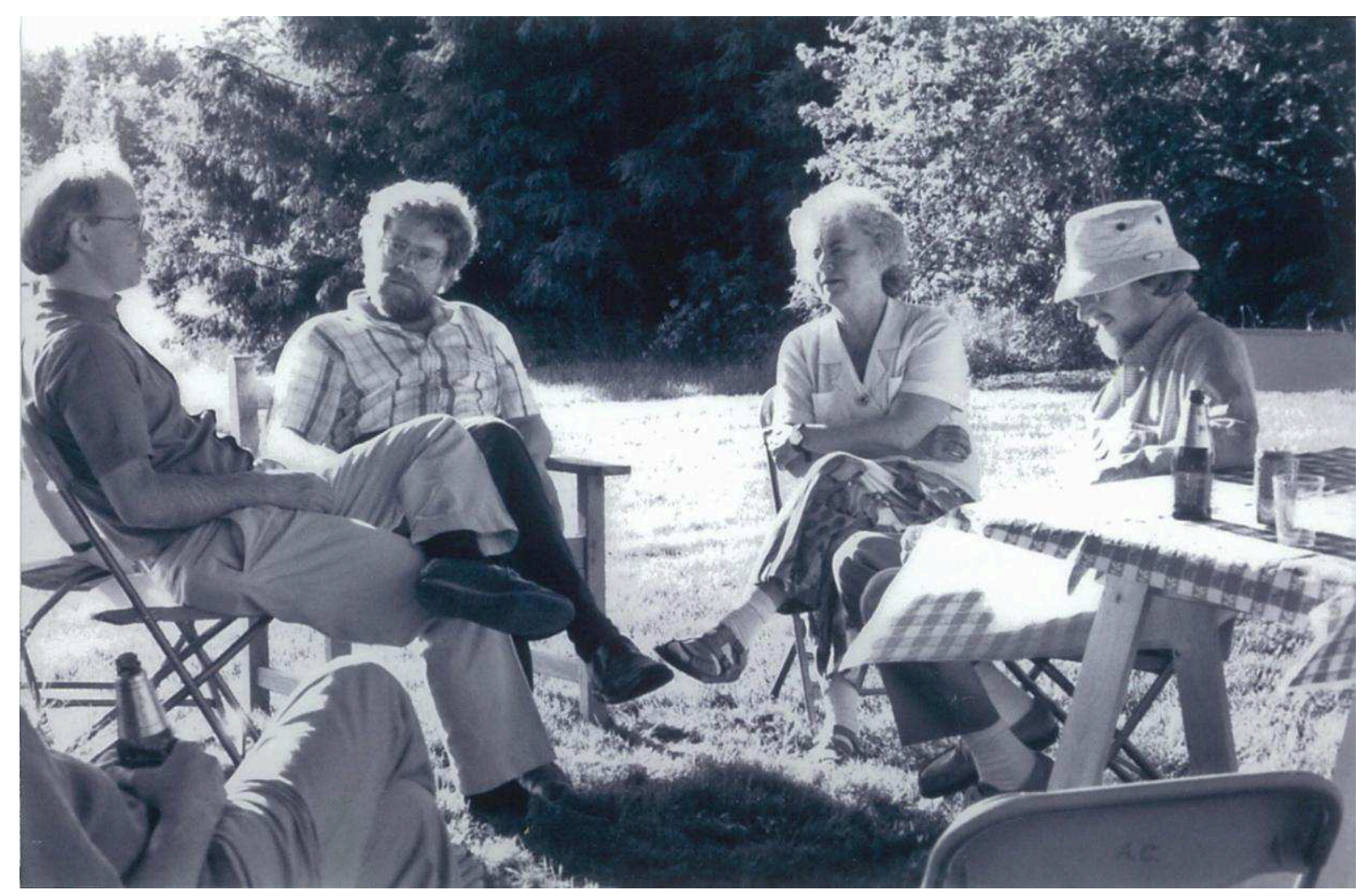

Fig. 1 - Arthur Zajonc, Anton Zeilinger, Mary Bell e John Bell, em uma conferência em Amherst, MA, 1990. Cortesia de Anton Zeilinger.

\section{Conteúdo e contexto}

Os dissidentes quânticos não defenderam a sua pesquisa em um vácuo. Se o ambiente profissional foi contra eles, desvalorizando suas pesquisas, outros fatores desempenharam um papel favorável. Em 1950, a Guerra Fria trouxe benefícios inesperados para os dissiden${ }^{10}$ H-D Zeh, Foundations of Physics, 1, 69-76 (1970). Rosenfeld to Jensen, 14 Fevereiro 1968, citado em The
Quantum Dissidents, op. cit., p. 306. Para a história completa, idem, p. 305-308. 
tes. Como parte das batalhas ideológicas da época, filósofos e físicos soviéticos criticaram a interpretação usual da mecânica quântica, ou seja, a complementaridade, por ser subjetivista. Ao contrário de outras críticas culturais, esta ressoou com críticas semelhantes feitas no Ocidente. Como cogitado pelo historiador da física, Max Jammer, o declínio da influência da interpretação da complementaridade pode ter sido fomentado e apoiado por movimentos sociais-culturais e fatores políticos, como o crescente interesse na ideologia marxista no Ocidente ${ }^{11}$. Esta afirmativa se mantém, apesar do fato de que havia físicos marxistas em ambos os lados da controvérsia, alguns favoráveis e outros contra o princípio de complementaridade de Bohr. No final dos anos 1950s e início dos anos 1960s, a velha guarda dos pais fundadores da física quântica estava se retirando do campo de batalhas filosóficas. A nova geração estava mais inclinada para criticar a interpretação usual da física quântica. Além disso, alguns da velha guarda entraram em confronto entre si, Rosenfeld defendendo o ponto de vista de Bohr e Wigner compartilhando a abordagem de von Neumann.

Ademais, Wigner entrou nessa disputa considerando-se um defensor da ortodoxia e acabou se considerando um heterodoxo. A monocracia da escola de Copenhague, para usar um termo de Jammer, quebrou-se por dentro. No final da década de 1960 e início da de 1970, a agitação social, política e cultural em torno da Guerra do Vietnã, forte nas universidades norte-americanas e europeias, levou os físicos a definir suas agendas de pesquisa abrindo-as para assuntos heterodoxos, tais como fundamentos. Na Itália, a Sociedade de Física Italiana promoveu fundamentos como um tópico bastante válido para as pesquisas na física, abrindo as portas de sua tradicional escola de verão, em Varenna, para o tema "fundamentos da mecânica quântica." D’Espagnat foi o diretor da escola enquanto Wigner proferiu a palestra de abertura apresentando a diversidade de soluções para o problema de medição. O teorema de Bell e os seus primeiros experimentos atraiu a atenção de muitos, incluindo Franco Selleri, que passou a dedicar grande parte de sua energia para o assunto. A Escola Varenna de 1970 foi o Woodstock de todos os dissidentes quânticos. De acordo com as lembranças de Anders Barany, então com 28 anos de idade, "o que mais me impressionou foi que muitos (talvez a maioria) dos cientistas altamente qualificados ensinavam na escola, mas não podiam cooperar para tentar ajudar os alunos a formarem uma imagem coerente dos diversos problemas relacionados com os fundamentos da física quântica" ${ }^{12}$. Do outro lado do Atlântico, tendências semelhantes estavam operando. Physics Today, a revista de prestígio da comunidade de físicos norte-americanos, promoveu editorialmente o debate sobre a interpretação da física quântica, abrindo-a com um artigo de DeWitt. Neste, a diversidade de interpretações possíveis foi apresentada e foi sustentada a interpretação de Everett. Na Califórnia, os físicos que seguiam a crescente contracultura hippie ficaram fascinados pelo emaranhamento e pelas possibilida-

\footnotetext{
${ }^{11}$ M. Jammer, The Philosophy of Quantum Mechanics - The Interpretations of Quantum Mechanics in Historical Perspective, Wiley, New York, 1974, p. 251.

12

The Quantum Dissidents, op. cit., p. 209.
} 
des aparentes de comunicação com velocidades maiores que a da luz. Esta ultima esperança não se confirmou, mas, no processo, boa física foi produzida ${ }^{13}$.

No início da década de 1980, particularmente após os experimentos de Alain Aspect sobre emaranhamento em 1982, a pesquisa em fundamentos da teoria quântica passou a ser considerada física de boa qualidade. A partir de meados da mesma década, avanços técnicos na manipulação de sistemas quânticos individuais e pesquisa aplicada facilitaram novos experimentos em fundamentos, tal como o experimento da escolha retardada proposto por John Wheeler ${ }^{14}$. Mais tarde, uma mescla da área de fundamentos com a ciência da computação selou o destino da pesquisa em fundamentos, a qual se tornou um dos pilares para o campo de pesquisa, em franco desenvolvimento, da informação quântica.

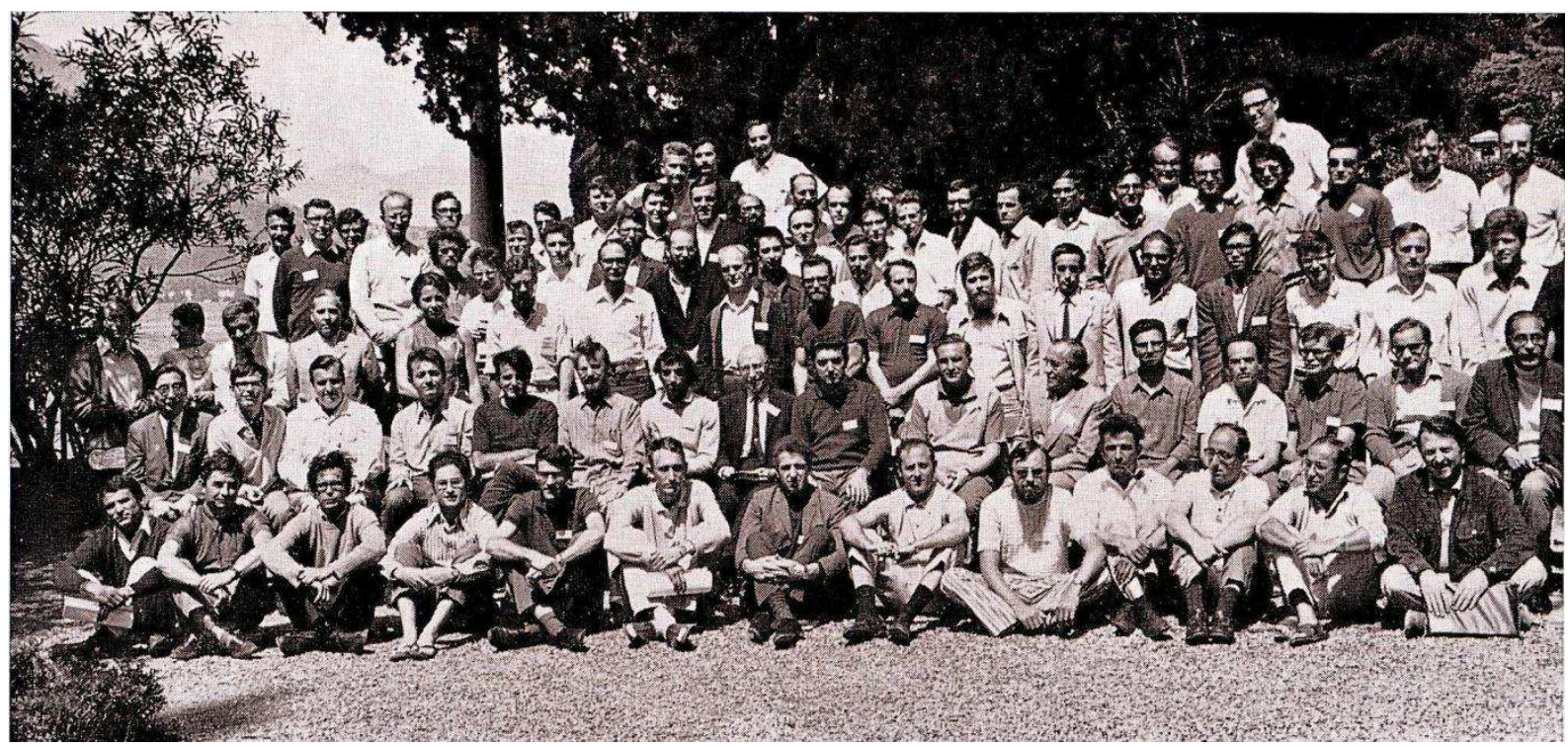

Fig. 2 - Participantes da Escola de Varenna, 1970, dedicada a fundamentos da mecânica quântica. Wigner está sentado no meio, o único com gravata. À sua direita, Sabbadini, e em seguida Bell. Reproduzido com permissão de "Foundations of Quantum Mechanics", Proceedings of the International School of Physics "Enrico Fermi", course IL, edited by B. d'Espagnant (Academic Press), (C) SIF, 1971.

\section{Conclusão}

Essa fascinante história nos mostra os muitos e variados fatores que condicionam o empreendimento cultural que chamamos de física. Esta narrativa não pode ser encerrada sem notarmos uma artimanha da história. Os dissidentes quânticos foram vitoriosos na medida em

\footnotetext{
13 Sobre os hippies, ver: D. Kaiser, How the Hippies Saved Physics: Science, Counterculture, and the Quantum Revival. W. W. Norton, New York, 2012.

J. Bromberg, Historical Studies in the Natural Sciences, 38(3), 325-352, 2008.
} 
que fundamentos tornou-se boa física. No entanto, eles tinham expectativas mais elevadas. John Bell, um verdadeiro ícone entre os físicos que trabalham com estas questões, costumava dizer que a mecânica quântica está "podre," citação da famosa frase de Hamlet, em uma referência oblíqua ao pai da interpretação padrão desta teoria, o físico dinamarquês Niels Bohr. A astúcia da história é que na tentativa de atacar os fundamentos da mecânica quântica, isto é, exibir limites na validade da teoria quântica, eles acabaram consolidando e desenvolvendo esta teoria física e a teoria quântica entrou no século 21 mais corroborada que nunca.

Esta história nos permite extrair conclusões que podem ser relevantes para uma reflexão sobre a natureza da ciência. A esta altura vamos apenas enunciar algumas destas conclusões, sem desenvolvê-las. Trata-se de uma história que nos permite valorizar uma leitura sociológica da mudança na ciência. $\mathrm{O}$ uso da noção de campo científico, na perspectiva de Pierre Bourdieu, ajuda-nos a compreender a estratégia dos dissidentes como uma estratégia de subversão (contraposição à estratégia de conservação) globalmente bem sucedida. Esta história nos mostra o valor da pluralidade na prática da ciência. A diversidade de interpretações da teoria quântica, sem resultados empíricos que permitam uma distinção entre estas interpretações, tem sido frutífera para o desenvolvimento da pesquisa em física. Trata-se também de um exemplo de imbricamento de contexto e conteúdo na mudança da ciência, evidenciando a limitação da dicotomia entre internalismo e externalismo na historiografia da ciência. Por fim, é uma história que nos revela uma lição filosófica, a da existência de conflitos contínuos, e produtivos, entre instrumentalismo e realismo na mudança da ciência. 